\title{
Räumlich-soziale Entmischung und die Finanzkrise der Kernstädte - das Beispiel Zürich
}

\section{André Odermatt, Zürich}

\section{Suburbanisation und städtische Finanzen}

Weltwirtschaftliche Umwälzungen, Globalisierung und Deregulierung gefährden zunehmend die Wettbewerbsfähigkeit der Städte. Die Position Zürichs im internationalen Standortwettbewerb ist an die effiziente und optimale Funktionsfähigkeit des Wirtschaftsraumes gebunden, der aus der Kernstadt und dem mit ihr verflochtenen Umland besteht. Die Funktionsfähigkeit der Stadt Zürich ist jedoch unter den gegenwärtigen Finanzproblemen immer weniger gewährleistet. Mit über 200 Millionen Franken weist die Stadt in der laufenden Rechnung 1996 zum siebten Mal in Folge einen hohen Ausgabenüberschuss aus. Eine dritte Erhöhung des Steuerfusses nach jenen der Jahre 1992 und 1993 kann wegen der interkommunalen Steuerkonkurrenz nicht erwogen werden. Daher konzipiert die Stadt Zürich seit mehreren Jahren Sparmassnahmen, die auch öffentliche Leistungen betreffen. Die aufsummierten jährlichen Einsparungen belaufen sich für das Jahr 1997 auf über 600 Millionen Franken oder ca. 10\% des Gesamtaufwands 1997. Dennoch hat sich das Bilanzdefizit bis Ende 1997 auf 1349 Millionen Franken erhöht. Mit vergleichbaren finanzpolitischen Problemen kämpfen auch andere schweizerische sowie ausländische Grossstädte.

Das Argument der ungünstigen Wirtschaftsstruktur der Kernstadt Zürich oder der seit 1991 anhaltenden Konjunkturschwäche ist kein ausreichender Erklärungsfaktor (ELSENER 1998: 7; JANOS, OdERMATT \& WaChTER 1997: 2). Die finanziell bedrängte Stadt Zürich ist Sitz zahlreicher wettbewerbsfähiger Unternehmen, die im wertschöpfungsintensiven und zukunftsträchtigen Segment der kommerziellen Dienstleistungen tätig sind. Aber auch junge, innovative high-techorientierte Kleinunternehmen etablieren sich an der Schnittstelle zwischen Forschung und Wirtschaft. Gegen das Argument der Konjunkturschwäche ist einzuwenden, dass kleinere Gemeinden, darunter die meisten Agglomerationsgemeinden der grossen Städte, ihre Finanzprobleme der frühen $90 \mathrm{er}$ Jahre unter Kontrolle bringen konnten. Die heutige Situation muss deshalb vielmehr als Folge des fortschreitenden Suburbanisationsprozesses und der damit verbunden funktionalen und sozialen Transformationsprozesse im urbanen Raum begriffen werden.

\section{Funktionale und soziale Entmischung als Ausgangspunkt}

Die heutige Situation der Kernstädte und ihrer Agglomerationen ist das Resultat einer Stadtentwicklung seit der Industrialisierung, die durch eine grundlegende räumliche Trennung von Wohnen und Arbeiten charakterisiert war und durch die Mobilitätszunahme und das dynamische $\mathrm{Be}$ völkerungswachstum der Agglomerationen seit den fünfziger Jahren verstärkt wurde (VETTIGER 1994: 27-28). Der schleichende Prozess der grossräumigen Trennung verschiedener alltäglicher Handlungsbereiche ist aber nicht abgeschlossen, sondern verändert kontinuierlich die räumliche Struktur der Agglomerationsräume. Neben dem weiteren Ausufern der Stadtlandschaft führt der Entmischungsprozess zu einer zunehmenden finanziellen Schwächung der Kernstadt. Funktionale und soziale Entmischung oder wirtschaftliche Spezialisierung ergeben sich aus der räumlichen Trennung sowie der räumlichen Differenzierung von Wirtschaft (Arbeitsstätten) und Bevölkerung (Wohnstätten) (VeTtiger 1994: 17). Einer der wichtigsten Gründe für die funktionale Entmischung ist der Grundrentenmechanismus (Janos, Odermatt \& Wachter 1997: 66-75). Höherwertige Nutzungen wie Dienstleistungen verdrängen schwächere Nutzungen, z. B. das Wohnen oder das Gewerbe, von den zentralen, gut erreichbaren Lagen des Agglomerationsraums. Zur funktionalen Entmischung können jedoch auch die Zonenplanung oder die Verkehrsinfrastrukturmassnahmen beitragen.

\subsection{Arbeitsplatz-Suburbanisation}

Die funktionale Entmischung führt zu einer wachsenden Zahl von Arbeitsplätzen in der Kernstadt bei gleichzeitigem Bevölkerungsverlust und einem kontinuierlichen Anstieg der Bevölkerung im sich laufend vergrössernden Agglomerationsraum. Die Entwicklung der funktionalen Entmischung zwischen 1970 und 1990 in der Agglomeration Zürich ist in der Tab. 1 aufgrund des Verhältnisses zwischen Beschäftigten und Wohnbevölkerung dargestellt.

Gesamtschweizerisch beträgt das Verhältnis von Beschäftigten zu Bevölkerung rund 1:2. In der gesamten Agglomeration Zürich (inkl. Stadt Zürich) ist das Verhältnis allerdings erheblich stärker zugunsten der Beschäftigten und beträgt rund 1:1,5. Dies kann als Indiz gewertet werden, dass der 


\begin{tabular}{|c|c|c|c|c|c|c|}
\hline & \multicolumn{3}{|c|}{$\begin{array}{c}\text { Beschäftigte } \\
\text { (gemäss Betriebszählung) }\end{array}$} & \multicolumn{3}{|c|}{ Wohnbevölkerung } \\
\hline & $\begin{array}{c}\text { absolut } \\
1990\end{array}$ & $\begin{array}{l}\text { in \% } \\
1990\end{array}$ & $\begin{array}{c}\text { Verän- } \\
\text { derung in } \\
\% \\
75-91 \\
\end{array}$ & $\begin{array}{c}\text { absolut } \\
1990\end{array}$ & $\begin{array}{l}\text { in \% } \\
1990\end{array}$ & $\begin{array}{c}\text { Verän- } \\
\text { derung in } \\
\% \\
70-90\end{array}$ \\
\hline Stadt Zürich & $357 ' 291$ & 55,6 & $+11,8$ & $365^{\prime} 043$ & 38,8 & $-13,6$ \\
\hline $\begin{array}{l}\text { Agglomeration (heutiges } \\
\text { Gebiet, ohne Stadt Zürich) }\end{array}$ & $285^{\prime} 445$ & 44,4 & $+49,0$ & $575^{\prime} 137$ & 61,2 & $+24,4$ \\
\hline Total & $642^{\prime} 736$ & 100,0 & $+26,9$ & $940^{\prime} 180$ & 100,0 & $+6,3$ \\
\hline
\end{tabular}

Tab. 1: Beschäftigte und Wohnbevölkerung in der Kernstadt und der Agglomeration

Employee and resident population in the central city and the suburbs

Emplois et population résidante dans la ville-centre et l'agglomération

Quelle: JANOS, OdermatT, WACHTER 1997: 32 (verändert).

Wirtschaftsraum Zürich deutlich über die statistisch definierte Agglomeration hinausreicht (Janos, OdermatT \& WaChTER 1997: 14). In der Stadt Zürich kommt auf eine wohnhafte fast eine beschäftigte Person. In den Agglomerationsgemeinden (ohne Stadt Zürich) beträgt das Verhältnis hingegen rund 1:2, entspricht also dem schweizerischen Durchschnitt. Der allgemeine Beschäftigungsüberschuss in der Agglomeration konzentriert sich somit fast ganz auf die Stadt Zürich. Allerdings muss erwähnt werden, dass der Zuwachs der Arbeitsplätze in den Agglomerationsgemeinden in den letzten 20 Jahren dynamischer verlaufen ist als in der Stadt Zürich. Die rezessiven 90er Jahre schlagen sich in einem deutlichen Rückgang der Beschäftigtenzahlen in der Agglomeration nieder. Im ganzen Agglomerationsgebiet inklusive der Stadt Zürich resultierte zwischen 1991 und 1995 ein Rückgang von 7,5\%. In derselben Zeitspanne verringerte sich die Beschäftigtenzahl in der Stadt Zürich um 11,2\%. Der grösste Teil der Arbeitsplätze ging also in der Stadt Zürich verloren (Statistisches Amt der StadT Zürich 1998: 129). Besonders verkehrstechnisch günstig gelegene Agglomerationsgemeinden weisen einen $\mathrm{Zu}$ wachs der Arbeitsplätze auf (ArbeitsplatzSuburbanisation). Vor allem die «Headquarter Economy» expandierte in den achtziger Jahren in die urbane Peripherie (Hitz, KeIL, Lehrer, RonneBerger, SChmid \& WOLFF 1995: 253-257). Somit bilden sich im Agglomerationsraum Nebenzentren aus, welche teilweise mit vergleichbaren Problemen wie die Kernstädte konfrontiert sind. Im Raum Zürich zeigt sich diese Entwicklung am deutlichsten im Norden rund um den Flughafen
Zürich Kloten, aber auch im Limmattal. Kloten als Standortgemeinde des Flughafens weist beispielsweise einen mit der Stadt Zürich vergleichbaren Beschäftigungsüberschuss aus.

\subsection{Reurbanisation}

Auch im Bereich der demographischen Entwicklung und des Wohnens zeigen sich neue Tendenzen: Stadtnahe Agglomerationsgemeinden sehen sich wie die Kernstadt aufgrund der demographischen Alterung zunehmend mit grösseren Anteilen älterer Bewohner konfrontiert und weisen als Folge davon leicht sinkende Einwohnerzahlen auf. In der Kernstadt manifestieren sich in jüngster Zeit Gentrifizierungsprozesse (KRÄTKE 1995: 177). Diese bausozialen Aufwertungsprozesse sind mit einer Verdrängung der ansässigen Bewohner durch einkommensstarke, oft jüngere Haushalte verbunden. Die Intensivierung von Gentrifizierungsprozessen ist ein bedeutendes Element der räumlich-sozialen Transformation prosperierender Grossstädte. Erfasst werden davon vor allem citynahe Wohnquartiere mit urbanen Wohnumfeldqualitäten. In Zürich sind dies beispielsweise das Industriequartier in Bahnhofsnähe oder das Seefeld in Citynähe. Die Aufwertung innerstädtischer Wohnviertel wird von Immobilienfirmen durch die Schaffung eines neuen urbanen Ambiente mit luxusmodernisierten Altbauten, postmodernen Neubauten als Lückenfüllern und innerstädtischen Einkaufszentren organisiert. Die Gentrifizierung ist also ein Teil der Reurbanisation, der Renaissance der Städte als Wohngebiete (FrEY 1990: 54). $\mathrm{Ob}$ damit aber die Bevölkerungsverluste der Vergangenheit wettgemacht werden können, ist 
fraglich. Der zusätzliche neue Wohnraum, der im Zuge der Renaissance der Städte als Wohngebiete und der Gentrifizierung geschaffen wurde, wird seit längerer Zeit durch die erhöhten Wohnflächenansprüche absorbiert. Dies zeigt sich auch in Zürich, wo sich der Wohnungsbestand zwischen 1990 und 1997 um knapp 4\% erhöhte - zwei Drittel davon mit 3 und mehr Zimmern - , die Bevölkerungszahl aber stagnierte (STATISTISCHES AMT DER STADT ZüRICH 1998/1: 1).

\subsection{Soziale Entmischung im Agglomerationsraum Zürich}

Soziale Entmischung bezeichnet die räumliche Differenzierung der Bewohner nach sozioökonomischen Merkmalen, z. B. Stellung im Lebenszyklus (Alter, Zivilstand, Haushaltstyp), die soziale Schichtzugehörigkeit (Bildung, berufliche Stellung, Einkommen) oder staatliche und kulturelle $\mathrm{Zu}$ gehörigkeit (Nationalität, Sprache, Religion). Bei der sozialen Entmischung spielt die Wohnstandortwahl eine wichtige Rolle, die gemäss der TieboutThese als vorteilhafte Kombination von «Versorgung mit öffentlichen Gütern» und «Steuerbelastung» angesehen werden kann. «Ein Haushalt wird bei rationaler Entscheidung jene Gemeinde als Wohnort wählen, deren Kombination aus öffentlichem Güterangebot (inklusive der natürlichen Umwelt) und Steuerbelastung ceteris paribus seinen Präferenzen am nächsten kommt» (РомMEREHNE 1987: 76). Das räumlich differenzierte Wohnraumangebot im Sinne objektivierter Möglichkeiten der Aneignung (Bourdieu 1987: 352, Odermatt 1997: 297-303) bildet die Voraussetzung, dass Haushalte überhaupt zwischen mehreren Orten wählen können, die jeweils Unterschiede in Höhe, Zusammensetzungen von öffentlichen Gütern und Leistungen und Steuern aufweisen.

Die Bedeutung des objektiven Wohnraumangebots für die räumlich-soziale Differenzierung zeigt sich beispielsweise in der Verteilung des Massenwohnungsbaus in der Agglomeration Zürich. Nach SснüTZ (1996) unterscheiden sich die Anteile aufgrund der unterschiedlichen Planungspraktiken zwischen den Gemeinden stark. Massenwohnungsbauten, in denen ein Haushalt das Treppenhaus mit mindestens 14 weiteren Haushalten teilt, prägen die Gemeinden Spreitenbach und Greifensee mit rund einem Drittel der Wohnungen und in absteigender Reihenfolge Schlieren, Dietikon, Opfikon, die Stadt Zürich und Kloten. Diese Gemeinden zeichnen sich heute durch ähnliche Bevölkerungsstrukturen wie die Stadt Zürich aus, insbesondere in bezug auf die Anteile der ausländischen Haushalte oder jener, die auf Sozialhilfe angewiesen sind.
Objektive Angebotsstrukturen beeinflussen auch die Abwanderung, vor allem jüngerer, mittelständischer bis wohlhabender und meist schweizerischer Familienhaushalte aus der Stadt Zürich (Meyrat Schlee \& Gafner 1998: 35). Ziel der Wohnstandortwahl sind immer entferntere, ländliche Gemeinden in der Peripherie der Agglomeration. Diese verfügen über einen relativ preisgünstigen Boden- und Immobilienmarkt, der die Erfüllung des Einfamilienhauswunsches ermöglicht. An den unattraktiven Wohnlagen, die aufgrund der abnehmenden Umweltqualität und der Verschlechterung des Wohnumfeldes vor allem in der Kernstadt, aber auch in gewissen stark belasteten Agglomerationsgemeinden zu finden sind, müssen die wirtschaftlich schwächeren Bevölkerungsschichten ihren Wohnungsbedarf decken. Die Wohnstandortverlagerungen führen somit im Agglomerationsraum Zürich zu einer kontinuierlichen sozialen Entmischung, weil vor allem mittlere bis hohe Einkommensklassen zwischen Steuerlast und Wohnqualität abwägen (können) und diese als ungleichgewichtig in der Kernstadt wahrnehmen. Dies fördert die Entwicklung hin zu sogenannten «A-Städten» (FREY 1996: 28), die durch überproportionale Anteile der Alten, Armen, Alleinstehenden, Auszubildenden, Ausländern, Arbeitslosen usw. geprägt sind.

Stellvertretend für die Indikatoren der Polarisierung zwischen Kernstadt und Agglomeration, wird hier die räumliche Verteilung der Haushalte nach Einkommensschichten dargestellt (Амт FüR Raumplanung des Kantons Zürich 1997: 25-26). Die grössten Anteile der Einkommensoberschicht (steuerbares Einkommen ab 170000 Fr.) weisen 1990 die Gemeinden am rechten und teilweise am linken Zürichseeufer sowie einige Gemeinden südwestlich von Zürich auf. Gemeinsames Merkmal dieser Agglomerationsgemeinden ist ihre Nähe zur Kernstadt. Umgekehrt weist die Stadt Zürich einen überdurchschnittlichen Anteil von Personen mit niederen Einkommen auf. Auch zwischen den verschiedenen Agglomerationsgemeinden kommt es zu Differenzierungen aufgrund der Bauzonierungspolitik oder aufgrund der spezifischen geographischen Lage, die z. B. die Lärmund Luftbelastung einer Gemeinde bestimmt. Die Agglomeration Zürich ist durch ein Süd-NordGefälle hinsichtlich der Einkommensverhältnisse, Anteile ausländischer Haushalte sowie anderer Merkmale gekennzeichnet (Janos, Odermatt \& WACHTER 1997: 220-225). Der soziale Hauptgegensatz liegt aber gleichwohl zwischen der Kernstadt und den Agglomerationsgemeinden.

Zusammenfassend kann gesagt werden, dass die fortschreitende Stadtentwicklung, verbunden mit den funktionalen und sozialen Entmischungs- 
erscheinungen, zu einem stark differenzierten, gleichzeitig aber auch vielfältig verflochtenen Zentrum-Umland-System führt. Die weitere Entwicklung wird dabei zunehmend von einer weiteren Polarisierung zwischen der Kernstadt und der Agglomeration, aber auch der Gemeinden der Agglomeration geprägt sein. In Anlehnung an den Begriff der vielfach geteilten Stadt (KRÄTKE 1995: 158) wird sich die Agglomeration zu einem vielfach geteilten und polarisierten urbanen Raum entwickeln.

\section{Folgen der funktionalen und sozialen Entmischung}

In Anlehnung an Frey (1996: 26-30) ergeben sich aus dem Entmischungsprozess vier sich selbst verstärkende Teufelskreise, die längerfristig die Wettbewerbsfähigkeit und die Lebensqualität der Städte, ihres Umlandes und der gesamten Schweiz gefährden. Die drei ersten Teufelskreise, die sich auf den Verkehr, die Umweltqualität und die Segregation zwischen den Gemeinden einer Agglomeration beziehen, führen auch zum vierten Teufelskreis, der sich auf die öffentlichen Finanzen und das Ungleichgewicht zwischen Kernstadt und Umland bezieht. Dabei liegt einer der Hauptgründe für die finanziellen Probleme der Stadt Zürich in der administrativ-politischen Unterteilung des Wirtschaftsraumes Zürich durch kommunale und kantonale Grenzen.

\subsection{Ausgaben der Gemeinden}

Die Gemeindeausgaben ergeben sich grundsätzlich aus einem spezifischen Finanzbedarf, der von der zentralörtlichen Stellung und der jeweiligen Bevölkerungsstruktur abhängig ist. $\mathrm{Zu}$ den zentralörtlichen Aufgaben gehören nach Voss (1991: 33-36) die:

- Versorgungsfunktion: Die Kernstädte versorgen nicht nur ihre Einwohner, sondern auch die ihres Verflechtungsbereiches mit privat und öffentlich angebotenen zentralörtlichen Gütern und Leistungen. Aus dieser regionalen Versorgungsfunktion erwächst dem zentralen Ort ein zusätzlicher, zentralitätsbedingter Finanzbedarf.

- Entwicklungsfunktion: Als Zentren mit einer hohen Dichte von Wirtschaftsaktivitäten geben sie Entwicklungsimpulse an das nähere und weitere Umland weiter. Diese Funktion findet ihre Rechtfertigung in den regionalen Entwicklungs- bzw. Wachstumstheorien.

In der räumlich eng verflochtenen schweizerischen Wirtschaft erbringen die Zentren zudem gewisse Aufgaben im überregionalen und teilweise sogar im landesweiten Interesse (ODERMatT 1998). Dazu zählen neben der wirtschaftlichen Innovations- und Entwicklungsfunktion beispielsweise Aufgaben im Bereich der Kultur oder des Sports. Neben dieser überregionalen und nationalen Bedeutung der Städte ist auf die hohe Dichte der Aktivitäten in den Kernstädten hinzuweisen. Wirtschaftliche Tätigkeiten, Wohnen und Verkehr sind auf engstem Raum konzentriert. Dies hat erhebliche «Kosten der Enge» zur Folge, die sich in einem hohen finanziellen Zusatzbedarf in den Bereichen Sicherheit, Verkehrsregulierung usw. bemerkbar machen. So betragen beispielsweise die Pro-KopfAusgaben in der Stadt Zürich für den Aufgabenbereich Rechtsschutz und Sicherheit ungefähr das $10 f a c h e$, im Bereich der Kultur und des Verkehrs je das über 25fache der Gemeinden im Kanton Zürich mit den jeweils tiefsten Ausgaben (JANOS, OdermatT \& WaChTER 1997: 197).

Theoretisch könnte davon ausgegangen werden, dass die Ausgaben einer Gemeinde weitgehend durch ihre Einkommensverhältnisse bestimmt würden. Wohlhabende Gemeinden hätten folglich ein gutes Versorgungsniveau mit öffentlichen Leistungen, während ärmere Gemeinden ein tieferes aufweisen würden. Aufgrund der engen funktionalen Verflechtung zwischen den Gemeinden in einem Agglomerationsraum und der kommunalen Steuerhoheit können die Gemeinden als «Trittbrettfahrer» handeln:statt höherer Ausgaben zur Hebung des örtlichen Leistungsniveaus können sie tiefere Steuerfüsse beschliessen. Als Berufs-, Freizeit-, Bildungs- und Einkaufspendler nutzen die Bewohner dieser Gemeinden weiterhin die zentralörtlichen Infrastrukturen der Kernstadt, führen wegen der Besteuerung am Wohnsitz jedoch keine Steuern direkt an die Leistungen der Kernstadt ab. Im räumlich-funktionalen Zusammenhang einer Agglomeration ist das Versorgungsniveau einer Gemeinde somit nicht nur vom lokalen Angebot abhängig, sondern auch von der Erreichbarkeit der öffentlichen Güter und Leistungen, welche in anderen Gemeinden, insbesondere in der Kernstadt, angeboten werden.

Im Unterschied zu nationalen öffentlichen Gütern, zum Beispiel der Landesverteidigung, bei der ein Ausweichen von potentiellen Nutzniessern kaum möglich ist, können sich Subjekte bei lokalen und/oder zentralörtlichen Kollektivgütern durch interkommunale Wanderung der Mitbeteiligung an der Finanzierung entziehen, ohne dass sie auf den Nutzen aus den öffentlichen Gütern und Leistungen der Nachbargemeinde oder der Kernstadt verzichten müssen. Daraus ergibt sich ein Zustand von Vorteilen ohne Nachteile für die Umlandgemeinden (Puchinger 1996: 119). Dieser ist dadurch gegeben, dass suburbane Gemeinden die 
positiven externen Effekte internalisieren und die kostenintensiven zentralen infrastrukturellen Ausgaben externalisieren. Die suburbanen Gemeinden verteidigen diesen Zustand so lange als möglich. Dadurch entsteht ein zirkulär-kumulativer Prozess, weil die Kernstadt zur Finanzierung öffentlicher Leistungen die Steuern anheben muss, vor denen die wohlhabenderen Bevölkerungsschichten wiederum in die steuergünstigen Umlandgemeinden entfliehen.

Dieser Prozess zeigt sich auch in der Agglomeration Zürich: Während die Stadt Zürich 1992 einen Steuerfuss von $123 \%$ aufwies, lagen die Steuerfüsse in den umliegenden Agglomerationsgemeinden zwischen $80 \%$ und $110 \%$ (JANOS, ODERMATT \& WACHTER 1997: 110-114). Zu den Gemeinden mit den tiefsten Steuerfüssen zählten die links- und rechtsufrigen Seegemeinden. Das Steuerfussgefälle zwischen Kernstadt und Umland hat sich seither weiter verstärkt. Die Kernstadt sieht sich also aufgrund ihrer zentralörtlichen Stellung mit einer hohen Ausgabenbelastung und einer hohen Steuerbelastung konfrontiert. Damit die öffentlichen Leistungen der Kernstadt für das Umland nicht in $\mathrm{zu}$ geringer Quantität und/oder Qualität angeboten werden, müsste die Stadt die Steuerbelastung weiter erhöhen. Dies ist politisch jedoch nicht durchsetzbar und würde zu einer Verschärfung der Steuerungerechtigkeit führen. Der Teufelskreis der öffentlichen Finanzen führt deshalb in einem Agglomerationsraum, der zwischen den Gemeinden unterschiedliche Besteuerungssätze kennt, zu einer suboptimalen Versorgung mit öffentlichen Leistungen. Die kommunale Steuerhoheit und der damit verbundene interkommunale Wettbewerb tragen aber auch zur Effizienzsteigerung bei der Bereitstellung öffentlicher Leistungen bei und erlauben, die allgemeine Steuerbelastung niedrig zu halten.

\section{Soziallasten}

Neben den zentralitätsbedingten Kosten ist auf die wachsenden sozialen Lasten der Kernstädte hinzuweisen. Als Folge der sozialen Entmischung haben die Kernstädte überproportionale Anteile wirtschaftlich schwächerer Bevölkerungsteile («AStadt»-Effekte). Attraktivitätsfaktoren sind vor allem die grössere Anonymität, die Hoffnung auf einen wirtschaftlichen Aufstieg sowie die zentralörtlichen Angebote im sozialen Bereich. Die Kernstädte werden so zu Kristallisationspunkten sozialer Probleme, deren Gründe oft auf nationaler Stufe zu suchen sind. So fallen beispielsweise die wesentlichen Entscheidungen zur Alters-, Arbeitslosenund Ausländerpolitik auf der nationalen und nicht auf der kommunalen Ebene. Die Tatsache, dass die Kernstädte den grössten Teil der Kosten der sozialen
Lasten tragen müssen, ist eine direkte Folge des Subsidiaritätsprinzips und der Aufgabenteilung zwischen Kanton und Gemeinden, welche wiederum durch die Aufgabenteilung zwischen Bund und Kantonen massgeblich bestimmt wird. Im Kanton Zürich lässt sich im Gegensatz zu einigen anderen Kantonen eine starke Tendenz der Abwälzung sozialer Kosten auf die Gemeinden feststellen. Die A-Stadt-Effekte führen als Folge davon zu übermässigen Belastungen der Stadt Zürich. Zur Verdeutlichung der Problematik kann die geographische Aufteilung der Bevölkerung und der Sozialleistungen im Kanton Zürich angeführt werden: Mit einem Bevölkerungsanteil von $29,5 \%$ entfallen auf die Stadt Zürich 50,5\% aller Bezüger von Sozialleistungen (Rüst 1995).

\subsection{Einnahmen der Gemeinden}

Die Folgen der funktionalen und sozialen Entmischung auf die Einnahmen der Stadt Zürich können durch die Aufteilung der in der Stadt Zürich verdienten Einkommen nach der Herkunft der Erwerbstätigen, basierend auf individuellen Personendaten aus der Volkszählung, vermittelt werden. Ausgehend von den Daten der Bundessteuerstatistik, in der die Reineinkommen der Steuerpflichtigen nach Gemeinden enthalten sind, wurde von Janos, Odermatt \& Wachter (1997: 62) als vorsichtige Schätzung angenommen, dass die Einkommen der nach Zürich pendelnden Erwerbstätigen dem jeweiligen Gemeindedurchschnitt entsprechen. Für die Agglomerationsgemeinden und die Gemeinden mit einem Pendlersaldo, also der Differenz zwischen Wegpendlern nach und $\mathrm{Zu}$ pendlern aus Zürich, von über 100 wurden die Einkommen gemeindeweise erfasst und ein neuer, mit der jeweiligen Pendlerzahl gewichteter Durchschnitt berechnet. Die übrigen Zupendlergemeinden wurden nicht gemeindeweise analysiert. Für die Schätzung der Einkommen der Pendler aus diesen Gemeinden wurde der Durchschnitt aller übrigen schweizerischen Gemeinden als Berechnungsgrundlage genommen. Daraus ergeben sich die in Tabelle 2 dargestellten Werte.

Insgesamt verdienten 1990 die 311897 Erwerbstätigen in der Stadt Zürich 17811123000 Fr. Durchschnittlich ergibt dies einen Betrag von 57106 Fr. pro Steuerpflichtigen. Ein bedeutender Teil des in der Stadt Zürich verdienten Reineinkommens wird aber durch die pendelnden Erwerbstätigen in ihre Wohnsitzgemeinden transferiert. Während die 151613 Erwerbstätigen mit Wohn- und Arbeitsort in der Stadt Zürich 48,6\% aller Erwerbstätigen ausmachen, beträgt ihr Anteil am gesamten Reineinkommen (8017447 Fr.) bloss 45,0\%. Die 110359 Erwerbstätigen mit Wohnsitz in der Agglomeration und Arbeitsort in der Stadt 


\begin{tabular}{|l|c|c|c|}
\hline $\begin{array}{l}\text { Wohnort der Erwerbstätigen gemäss } \\
\text { Volkszählung in der Stadt Zürich }\end{array}$ & $\begin{array}{c}\text { Zupendler/ } \\
\text { Erwerbstätige }\end{array}$ & $\begin{array}{c}\text { Reineinkommen pro } \\
\text { Pflichtige, Fr. }\end{array}$ & $\begin{array}{c}\text { Reineinkommen 1'000 } \\
\text { Fr. }\end{array}$ \\
\hline $\begin{array}{l}\text { Agglomerationsgemeinden im Kanton } \\
\text { Zürich }\end{array}$ & $103^{\prime} 666$ & $65^{\prime} 980$ & $6^{\prime} 839^{\prime} 929$ \\
\hline $\begin{array}{l}\text { Agglomerationsgemeinden ausserhalb des } \\
\text { Kantons Zürich }\end{array}$ & $66^{\prime} 693$ & $60^{\prime} 120$ & $402^{\prime} 381$ \\
\hline $\begin{array}{l}\text { Agglomeration } \\
\text { (ohne Stadt Zürich) }\end{array}$ & $110^{\prime} 359$ & $65^{\prime} 625$ & $7^{\prime} 242^{\prime} 310$ \\
\hline Gemeinden mit Pendlersaldo $\left.{ }^{3} 100\right)$ & $20^{\prime} 513$ & $52^{\prime} 952$ & $1^{\prime} 086^{\prime} 207$ \\
\hline Übrige Zupendler (Schweiz) & $29^{\prime} 412$ & $49^{\prime} 815$ & $1^{\prime} 465^{\prime} 159$ \\
\hline Zupendler insgesamt & $160^{\prime} 284$ & $61^{\prime} 102$ & $9^{\prime} 793^{\prime} 676$ \\
\hline $\begin{array}{l}\text { Erwerbstätige mit Wohn- und Arbeitsort } \\
\text { in der Stadt Zürich }\end{array}$ & $151^{\prime} 613$ & $52^{\prime} 881$ & $8^{\prime} 017^{\prime} 447$ \\
\hline Enwerbstätige in der Stadt Zürich & $311^{\prime} 897$ & $57^{\prime} 106$ & $17^{\prime} 811^{\prime} 123$ \\
\hline
\end{tabular}

Tab. 2: Reineinkommen in der Stadt Zürich 1990 (Schätzung)

Net income in the City of Zurich 1990 (estimation)

Revenu dans la ville de Zurich 1990 (estimation)

Quelle: JANOS, ODERMATT, WACHTER 1997: 63 (verändert).

Zürich, welche $35,4 \%$ aller Erwerbstätigen in der Stadt Zürich ausmachen, transferieren $40,7 \%$ (7242310 Fr.) der in der Stadt Zürich verdienten Einkommen in ihre Wohnsitzgemeinden. Dort versteuern sie ihr Einkommen oft zu tieferen Steuersätzen als in der Stadt Zürich. Der überdurchschnittliche Einkommensanteil der Erwerbstätigen mit Wohnsitz in der Agglomeration ist eine Folge der höheren Reinkommen der Steuerpflichtigen in der Agglomeration. Das durchschnittliche Reineinkommen ist mit 65625 Fr. deutlich höher als dasjenige von 52881 Fr. der Erwerbstätigen mit Wohn- und Arbeitsort in der Stadt Zürich. Der Grund dafür ist wiederum in der sozialen Entmischung zu suchen. Als Beispiel können die einkommensstarken Berufskategorien der Direktoren und leitenden Angestellten angeführt werden: Von den knapp 28000 Direktoren und leitenden Angestellten, die in der Stadt Zürich tätig sind, wohnen fast $70 \%$ in der Agglomeration (JANOS, ODERMATT \& WACHTER I997: 43). Insgesamt fliessen $55 \%$ der in der Stadt Zürich verdienten Reineinkommen - ohne dass dieses für die Stadt Zürich steuerlich erfassbar wäre - in die Wohnsitzgemein- den der 160284 Pendler, was in ähnlicher Weise auch für Bern bestätigt wurde (GÄcHTER \& HoFMANN 1996).

\section{Einnahmen von juristischen Personen}

Oft wird argumentiert, dass die Kernstädte ihren zentralörtlichen Finanzbedarf mit den Steuereinnahmen von den juristischen Personen abdecken können, da sie aufgrund der funktionalen Entmischung über eine hohe Zahl besteuerbarer Unternehmen verfügen. In der Stadt Zürich ist der Einnahmenanteil aus Unternehmenssteuern tatsächlich erheblich höher als in den Agglomerationsgemeinden oder im Kanton. Dies drückt sich auch in einer hohen Steuerkraft pro Kopf der Bevölkerung aus. Hierbei bezeichnet die Steuerkraft den auf die Einwohnerzahl bezogenen Ertrag der allgemeinen Gemeindesteuern von natürlichen und juristischen Personen umgerechnet von den zur Anwendung gelangten Steuerfüssen auf $100 \%$. Die Stadt Zürich lag 1992 mit einer Steuerkraft von 3070 Fr. leicht über dem kantonalen Durchschnitt. Als Vergleich dazu weisen die vier steuerkräftigsten Gemeinden des Kantons Zürich (Zollikon, 
Küsnacht, Zumikon und Uitikon) aber eine Steuerkraft von über 5000 Fr. bis knapp 7400 Fr. auf (JANOS, ODERMATT \& WaChTER I997: 158f.).

Bei guter Wirtschaftslage kann die Stadt Zürich mit den Einnahmen von den Unternehmen durchaus einen beträchtlichen Anteil der zentralörtlich bedingten Ausgaben decken. Die Höhe der Einnahmen von den juristischen Personen ist jedoch sehr stark von der jeweiligen wirtschaftlichen Lage abhängig. Konjunktureinbrüche machen sich bei Unternehmen im Gegensatz zu den natürlichen Personen rasch bemerkbar. So ist beispielsweise der jährliche Gemeindesteuerertrag der Grossbanken zu $100 \%$ in der Stadt Zürich von 97 Millionen Franken (1994) auf 21 Millionen Franken (1997) gesunken (STADTRAT ZüRICH 1998: 11). Zudem ist zu berücksichtigen, dass sich die Steuerkraft definitorisch ausschliesslich auf die Einwohner einer Gemeinde bezieht und beispielsweise nicht auf die Summe der Einwohner und Arbeitsplätze. Diese in der Finanzkraftstatistik oft verwendete Vergleichsgrösse geht somit von der Fiktion aus, dass Arbeitsplätze nur Erträge bringen, aber keinen durch Steuermittel zu finanzierenden Aufwand verursachen. Dass dies nicht der Fall ist, zeigt sich offensichtlich beim Verkehr, und zwar im öffentlichen Verkehr wie auch im Privatverkehr. In Zeiten langwieriger Rezession oder hartnäckiger Strukturprobleme können die Kernstädte deshalb in ernsthafte Schwierigkeiten geraten (FREY 1990: 297).

\subsection{Möglichkeiten der Ausgabenreduktion}

Es bleibt die Frage zu beantworten, weshalb bei sinkenden Einnahmen die Kernstädte nicht einfach ihre Ausgaben reduzieren. Aus den bisherigen Ausführungen ergeben sich mehrere Gründe, weshalb sinkende Steuereinnahmen nicht automatisch zu sinkenden Staatsausgaben führen (JANOS, OdermatT \& WaChTER 1997: 19f.):

- In der zentralen Gemeinde bleiben die Ansprüche der eigenen Bevölkerung an den Staat unverändert hoch oder steigen aufgrund der einseitigen Bevölkerungsstruktur sogar an. Aufgrund der hohen Konzentration von Arbeitsplätzen in der Kernstadt verringert sich auch der Infrastrukturbedarf nicht.

- Zentralörtliche Güter und Leistungen werden in den unterschiedlichsten Bereichen von der gesamten Agglomerationsbevölkerung nachgefragt.

- Die funktionale und soziale Entmischung führt zu negativen Ballungseffekten, wie Verkehrsstaus, Kriminalität usw., was einen hohen Regulierungsaufwand induziert.

- Der Abbau bestehender öffentlicher Leistungen ist mit wirtschaftlichen und politischen Kosten verbunden.
Wird der Druck durch das städtische Defizit so stark, dass Gegenmassnahmen ergriffen werden müssen, so führt dies zu höheren Steuerfüssen oder zu einer Senkung der Staatsausgaben. Diese erfolgen aber vor allem im «Wunschbereich» der öffentlichen Leistungen. Durch den Abbau öffentlicher Leistungen in der Kernstadt wird aufgrund der versorgungspolitischen Bedeutung und der Entwicklungsfunktion der Kernstadt aber das gesamte Agglomerationssystem geschwächt. Der funktional stark verflochtene Siedlungs- und Wirtschaftsraum Zürich ist insgesamt gesehen also durch ineffiziente und suboptimale Strukturen sowie ungerechte Steuerverhältnisse gekennzeichnet.

\section{Folgerungen}

Gut funktionierende Kernstadt-Umland-Beziehungen sind von grundlegender Bedeutung für die wirtschaftliche und soziale Funktionserfüllung der Agglomerationen innerhalb der Schweiz, aber auch für die Sicherung der internationalen Wettbewerbsfähigkeit der Schweiz als Ganzes. Die oben dargestellten ineffizienten, suboptimalen Strukturen im Raum Zürich müssen deshalb dringend verbessert werden.

\subsection{Räumliche Verflechtungen und fiskalische Äquivalenz}

Die Analyse zeigte auf, dass die Agglomerationen als funktional eng verflochtene Räume betrachtet werden müssen. Im föderalistischen Staatsaufbau der Schweiz machen sich jedoch administrative Grenzen als teilendes Element bemerkbar. Der Hauptgegensatz liegt dabei zwischen der Kernstadt, welche die Zentrumsfunktionen wahrnimmt, und den Umlandgemeinden, welche Nutzniesser der zentralörtlich angebotenen Güter und Leistungen sind, diese aber nur ungenügend abgelten. Im heutigen Kernstadt-Umland-System entstehen folglich sogenannte Spill-overs. Spill-overs sind räumliche externe Effekte und beziehen sich auf Leistungen, die über die politischen Gebietsgrenzen hinweg ausgetauscht werden. Es sind positive oder negative Auswirkungen einer Gebietskörperschaft (Kantone, Gemeinden) auf die Einwohner oder Unternehmungen anderer Gebietskörperschaften, für welche keine Abgeltung bezahlt wird (FREY 1990: 285).

Ein räumliches System, in dem Spill-overs auftreten, verletzt das regionalwirtschaftliche Postulat der fiskalischen Äquivalenz. Gemäss Meier (1996: 66) besagt das theoretische Prinzip der Äquivalenz, dass die Bereitstellung öffentlicher Leistungen dann optimal ist, wenn diejenigen, die den Nutzen einer Leistung haben, über diese Leistung ent- 
scheiden und dafür zahlen. Fiskalische Äquivalenz ist dann erreicht, wenn alle Nutzen und Kosten internalisiert sind. Im heutigen Agglomerationssystem können die Umlandgemeinden der Stadt Zürich aber als «Trittbrettfahrer» handeln (JANOS, Odermatt \& WachTER 1997: 184 f.). «Wenn die partizipierenden Umlandgemeinden damit rechnen können, dass sich ihre Einwohner auch ohne Finanzbeiträge in den zentralen Orten versorgen können, werden sie rationalerweise nicht zu einem finanziellen Engagement bereit sein» (Voss 1991: 274). Weil bei grenzüberschreitenden Leistungen die Kreise der Entscheidungsträger, der Kostenträger und der Nutzniesser auseinanderfallen, treten zudem Leistungsansprüche und -angebote auf, die nicht bedarfsgerecht sind. Wenn also Nutzniesser über das von ihnen genutzte Angebot nicht mitentscheiden und wenn sie dafür nicht die wahren Kosten bezahlen, dann entspricht die Nachfrage nicht mehr einem echten Bedürfnis. Es werden zuwenig oder zuviel Leistungen produziert und konsumiert. Öffentliche Leistungen sind als Folge davon entweder zu teuer oder nicht bedarfsgerecht.

\subsection{Allokative Effizienz}

Insgesamt gesehen ist also die allokative Effizienz des Agglomerationssystems nicht mehr gegeben. Gemäss Voss (1991: 46) kann diese wie folgt umrissen werden: Das volkswirtschaftliche Ziel der Allokationseffizienz (sog. Pareto-Effizienz) bezieht sich auf einen Gesamtzustand der volkswirtschaftlichen Güterversorgung. Die Effizienz hat dabei zwei Komponenten:

- Bei gegebenem Ressourcenbestand und gegebener Produktionstechnik soll ein maximales gesamtwirtschaftliches Gütervolumen erzeugt werden.

- Die Struktur des gesamtwirtschaftlichen Gütervolumens soll mit den individuellen Nachfragestrukturen der Konsumenten übereinstimmen.

Die allokative Effizienz kann nur dann erreicht werden, wenn die Aufgaben der öffentlichen Hand effizient erfüllt werden. Dies bedingt eine kostenminimale Produktion und die Einhaltung des Prinzips der fiskalischen Äquivalenz. Eine Agglomeration ist dann effizient, wenn die gemeindeinternen Verfahren der Leistungserstellung produktiv sind und die zentralörtlichen Aufgaben sich auf die Präferenzen der eigenen Einwohner als auch auf die mitzuversorgenden Umlandeinwohner beziehen (Voss 1991: 47). Im heutigen System wird das allokative Effizienzziel nicht erreicht.

\subsection{Spill-over-Effekte}

Spill-overs erzeugen zudem Gefühle der Ungerechtigkeit bei den Steuerzahlern von Gemeinwesen, welche zentralörtliche Leistungen anbieten (FREY 1990:285). Sie tragen Kosten für Dienste, von denen andere unentgeltlich profitieren - und fühlen sich ausgebeutet. Aufgrund der funktionalen und sozialen Entmischung muss eine immer kleinere und durchschnittlich gesehen wirtschaftlich schwächere Zahl von Personen die zentralörtlichen Lasten tragen. Das heutige Steuersystem (Schwerpunkt bei der Besteuerung der Einwohner nach dem Wohnsitzprinzip) bewirkt für die Kernstädte eine immer grösser werdende Lücke zwischen dem zentralörtlichen Finanzbedarf und den Steuereinnahmen. Somit wird mit den heutigen Steuerverhältnissen in der Agglomeration Zürich auch ein allgemein anerkannter Steuergrundsatz verletzt, wonach zwei Subjekte in gleicher Position ungeachtet der geographischen Lokalisation ihres Wohnsitzes gleich besteuert werden sollen, sofern der Nutzen aus geleisteten Steuern für beide gleich hoch ist (Book 1970: 168 f.). Innerhalb der versorgungspolitisch verflochtenen Agglomeration ist dieser Grundsatz aber verletzt, denn die verschiedenen Subjekte können durch die Lokalisation ihres Wohnsitzes einen Steuervorteil erzielen.

Da die Spill-overs den kommunalen Wettbewerb verzerren und die weitere Segregation der Steuerzahlenden vorantreiben, sollten sie im Hinblick auf die Konsolidierung und Verbesserung der Funktionsfähigkeit der Stadt Zürich und der Agglomeration bereinigt werden (ELSENER 1998: 121). Das grundsätzliche Ziel von Lösungsvorschlägen formulieren FREY \& LANGLOH (1995: 147) wie folgt: Die Kreise der Nutzniesser, der Entscheidungsträger und der Kostenträger von öffentlichen Leistungen sollen wieder besser zur Deckung gebracht werden. Ziel ist die Herstellung fiskalischer Äquivalenz: Wer als Region oder Gemeinde profitiert, soll zahlen, und wer zahlt, soll mitentscheiden können. Es geht also um Massnahmen zur Herstellung eines gerechteren Agglomerationssystems. Der Konkurrenzkampf zwischen den Agglomerationsgemeinden, bei dem mit möglichst tiefen Steuern wohlhabende Leute angezogen werden sollen, könnte dadurch vermindert werden.

\subsection{Horizontaler und vertikaler Lastenausgleich}

Eine Lösung des Spill-over-Problems bietet in erster Linie ein horizontaler Lastenausgleich zwischen den Gemeinden der Agglomeration und der Kernstadt. Für gewisse zentralörtliche Leistungen und die Lasten, welche durch die einseitige Bevölkerungs- und Wirtschaftsstruktur entstehen, sollten auch gezielte vertikale Abgeltungen in Betracht gezogen werden. 
Mit dem in der Abstimmung vom 7. Februar 1999 gewährten Lastenausgleich für bestimmte Aufgabenbereiche zugunsten der Stadt Zürich ist ein erster Schritt zu gerechteren Agglomerationsstrukturen im Kanton Zürich getan. Gewisse Aufgaben (z. B. Polizei, Soziallasten) werden aber nur für eine Übergangszeit abgegolten und bedürfen in Zukunft einer grundsätzlichen Lösung. Ausgleichsmechanismen sollten auch kantonsübergreifend institutionalisiert werden, da die Agglomerationen wie im Fall von Zürich die Kantonsgrenzen teilweise überschreiten. Aufgrund der nationalen Ausstrahlung der Kernstädte ist für gewisse Leistungen der Städte, die sie in einem nationalen Interesse erbringen, auch ein Engagement des Bundes denkbar (Odermatt 1998). In Frage kommen beispielsweise Kultureinrichtungen (ElSENER 1998) und Sportanlagen mit nationaler und internationaler Ausstrahlung oder Leistungen, welche die Städte in ihrer Funktion als nationale Verkehrsdrehscheiben wahrnehmen.

Neben dem horizontalen und vertikalen Finanzausgleich sollten auch andere Massnahmen zur Verbesserung der Funktionsfähigkeit der Kernstädte innerhalb des Agglomerationssystems konzipiert werden, beispielsweise Steuerausscheidungsmodelle zwischen Wohnsitz und Arbeitsplatzgemeinden. Bereits heute bestehen Vereinbarungen über eine solche Steuerausscheidung für die Grenzgänger in den Kantonen Basel und Genf. Da die Agglomerationen vor den Kantonsgrenzen nicht haltmachen, ist mittelfristig auch über eine Steuerharmonisierung auf Bundesebene zu diskutieren, welche die Steuerkonkurrenz mildert, den Steuerwettbewerb zwischen den Gemeinden aber nicht gänzlich unterbindet. Auch neue Formen der Zusammenarbeit in den Agglomerationen müssten erwogen werden. Ein Beispiel ist der neu ins Leben gerufene Agglomerationsverbund Freiburg. Ein anderer Vorschlag geht in Richtung der Etablierung von Funktionalregionen, die sich nach der optimalen Betriebsgrösse einer öffentlichen Aufgabe richten.

In diesem Beitrag wurde aufgezeigt, dass die funktionale und soziale Entmischung als Grund der heutigen Probleme der Kernstädte betrachtet werden muss. Es genügt deshalb nicht, nur die Symptome der polarisierten Strukturen zu lindern. Vielmehr brauchte es neue Ansätze für die Planung des Agglomerationsraumes. In Einklang mit dem AmT FÜr RAUMPLANUNG DES Kantons ZÜRICH (1997: 2) kann postuliert werden, dass die Raumordnungspolitik zur Lösung räumlich-sozialer Ungleichgewichte einen substantiellen Beitrag leisten müsste, indem sie planerisch in den kontinuierlichen funktionalen und sozialen Entmischungsprozess innerhalb der Agglomeration eingreift. Dies bedingt die Einschränkung von Immissionen, die Umzonung bestimmter Gebiete, die Schaffung von Frei- und Grünräumen, die Realisierung nachgefragter Infrastruktureinrichtungen sowie eine überregionale Wohnpolitik. Nur so kann die Polarisierung vermindert und das im Planungs- und Baugesetz des Kantons Zürich festgelegte Ziel der gleichwertigen Lebensbedingungen und der sozialen Durchmischung erreicht werden.

\section{Literatur}

AMT Für Raumplanung DES Kantons Zürich (1997): Sozial-räumliche Durchmischung. Raumbeobachtung Kanton Zürich 19.

Book, S. H. (1970): Costs of Commuters to the Central City as a Basis for Commuter Taxation. Ann Arbor - London.

BourdiEu, P. (1987): Die feinen Unterschiede. Kritik der gesellschaftlichen Urteilskraft. Frankfurt a. M. ElSENER, M. (1998):Ausgebeutete Kernstadt? Spillovers im subventionierten Kulturangebot der Stadt Zürich am Beispiel des Schauspielhauses und des Kunsthauses Zürich. Diplomarbeit am Geographischen Institut der Universität Zürich. Zürich.

FrEY, R. L. (1990): Städtewachstum, Städtewandel. Basel.

FREY, R. L. \& P. M. LANGLOH (1995): Basel plus. Ökonomische Grundlagen für eine auf die Agglomeration ausgerichtete Stadtentwicklung. Basel.

GäCHTER, E. \& W. HOFMANN (1996): Arbeitspendler, Arbeitseinkommen und Steuerströme. Studie Zentrumslasten und Zentrumsnutzen. Amt für Statistik und Präsidialdirektion der Stadt Bern. Bern.

Hitz, H., Keil, R., Lehrer, U., Ronneberger, K., Schmid, C. \& R. WolfF (1995): Capitales Fatales. Urbanisierung und Politik in den Finanzmetropolen Frankfurt und Zürich. Zürich.

Janos, E., Odermatt, A. \& D. Wachter (1997): Sozioökonomische Strukturen im Raum Zürich. Soziale und funktionale Entmischung im Raum Zürich: Ursachen, Probleme, Handlungsmöglichkeiten. - = Wirtschaftsgeographie und Raumplanung, 24 - Zürich.

KRÄTKE, S. (1995): Stadt - Raum - Ökonomie. Einführung in aktuelle Problemfelder der Stadtökonomie und Wirtschaftsgeographie. - = Stadtforschung aktuell, 53 - Basel, Boston, Berlin.

Meier, A. (1996): Das heutige System des bundesstaatlichen Finanzausgleichs. Eine kritische Betrachtung aus Sicht der Wissenschaft. - In: MäDER, H. \& K. SCHEDler (Hrsg.): Perspektiven des Finanzausgleichs in der Schweiz. Bern, Stuttgart, Wien: 65-77.

Meyrat Schlee, E. \& A. Gafner (1998): Soziale Entmischung in der Stadt Zürich. Studie im Auftrag 
der Fachstelle für Stadtentwicklung. Zürich. OdERMATT, A. (1997): Eigentümerstrukturen des Wohnungsmarktes. Ein handlungstheoretischer Beitrag zur Erklärung der räumlich-sozialen Wohnstandortverteilung am Fallbeispiel Schweiz. Münster.

Odermatt, A.. (1998): Die Bundesstaatliche Finanzordnung aus Sicht der Städte und Agglomerationen. - = Wirtschaftsgeographie und Raumplanung, 27. Zürich.

POMMEREHNe, W. W. (1987): Präferenzen für öffentliche Güter. Tübingen.

PuCHINGRT, K. (1996): Das Agglomerationsdilemma - Vorteile ohne Nachteile. - In: DÖRR, H. (Hrsg.): Interkommunale Kooperation in Stadtregionen. Salzburg/Wien: 117-124.

RüsT, H. (1995): Sozialbericht Kanton Zürich 1994, Fürsorgedirektion des Kantons Zürich. Zürich.

SchÜTZ, M. (1996): Massenwohnungsbau in den 60er und 70er Jahren in der Agglomeration Zürich. Diplomarbeit am Geographischen Institut der Universität Zürich. Zürich.

STADTRAT ZüRICH (1998): «Leistungsabgeltung.doc.» Dokumentation des Zürcher Stadtrates zur kantonsrätlichen Vorlage der Leistungsabgeltung. Zürich. Statistisches Amt Der STAdT ZürICH (1998): Statistisches Jahrbuch der Stadt Zürich. Zürich.

Statistisches Amt Der Stadt Zürich (1998/1): Gebäude- und Wohnungsbau 1997. Gebäude- und Wohnungsbestand Ende 1997. - = Zürcher Statistische Nachrichten 1.

VETTIGER, H. (1994): Lebensverhältnisse und Lebensqualität in städtischen Räumen der Schweiz - Möglichkeiten der Anwendung objektiver und subjektiver Raumbeobachtungsindikatoren. Dissertation der HSG, Nr. 1509. Bamberg.

Voss, W. (1991): Nutzenspillover-Effekte als Problem des kommunalen Finanzausgleichs. Ein Beitrag zur ökonomischen Rationalität des Ausgleichs zentralitätsbedingten Finanzbedarfs. Bern, Frankfurt a. M.

\section{Zusammenfassung: Räumliche-soziale Entmischung und die Finanzkrise der Kernstädte - das Beispiel Zürich}

Im Kontext der funktionalen und sozialen Entmischung im Agglomerationsraum wird die finanzielle Krise der Kernstädte am Beispiel der Stadt Zürich thematisiert. Die zunehmende Polarisierung zwischen Kernstadt und Agglomeration führt zu einem Ungleichgewicht zwischen den Einnahmen und Ausgaben der öffentlichen Hand in der Kernstadt. Durch die Tatsache, dass die pendelnden Erwerbstätigen ihre Einkommen aus der Kernstadt in die Wohnsitzgemeinden transferieren, erodieren die Steuereinnahmen der Stadt, während die Infrastrukturausgaben hoch bleiben. Das Agglomerationssystem weist als Folge davon ineffiziente Strukturen auf, und die Wettbewerbsfähigkeit der Stadt Zürich ist dadurch zunehmend gefährdet. Neben der Lösung des «Spill-overProblems» und der Abgeltung der durch die «AStadt-Effekte» bedingten steigenden Soziallasten müssen längerfristig raumplanerische Massnahmen zum Abbau der polarisierten Agglomerationsstrukturen in Erwägung gezogen werden.

\section{Résumé: La ségrégration fonctionelle et sociale et la crise financière des villes-centres - l'exemple de la ville de Zurich}

L'article présent traite le sujet de la crise financière des villes-centres à l'exemple de la ville de Zurich dans le contexte de la ségrégation fonctionnelle et sociale dans l'agglomération. La polarisation accrue entre la ville-centre et ses communes adjacentes engendre un déséquilibre entre les recettes et les dépenses du budget public dans la villecentre. Du fait que les travailleurs pendulaires transfèrent leurs revenues depuis la ville-centre vers les municipalités suburbaines, les recettes de la ville-centre diminuent. En même temps, les dépenses restent au même niveau car les pendulaires bénéficient de l'infrastructure de la ville-centre. Il en résulte un système métropolitain qui affiche des structures inefficaces. La capacité d'entrer en concurrence de l'agglomération de Zurich avec d'autres agglomérations se voit ainsi de plus en plus menacée. Il faudrait donc, à long terme, non seulement résoudre le problème du «spill-over» et de la péréquation des charges sociales dues aux effets des structures de population dans la ville-centre mais également prendre en considération des mesures de planification permettant de diminuer les structures d'agglomération polarisées.

\section{Summary: Functional and social segregation processes and the fiscal crisis of central cities - the case of Zurich}

This paper explores the financial crisis of the central city as a result of the functional and social segregation between the city and the suburban areas, using Zurich as an example. The increasing central city-suburb disparity leads to an imbalance between the income and expenditures of the central city budget. Due to the transfer of income by commuters and the fact that taxes on income earned in the central city are levied at the place of residence the central city tax base erodes. Costs remain quite high due to the maintenance of the city's infrastructure. As a result, the metropolitan system is characterized by inefficiencies. This increasingly jeopardizes the competitive position of the entire metropolitan system. A solution is 
needed to solve the problem of insufficient city finances. Apart from the immediate solution to the "spill over" problem, longer-term planning measures must be considered to reduce the continued polarization of metropolitan areas.

Dr. phil. André Odermatt, Geographisches Institut, Universität Zürich, Abteilung Wirtschaftsgeographie, Winterthurerstrasse 190, CH-8057 Zürich. 